\title{
Multiobjective sensitivity analysis and optimization of distributed hydrologic model MOBIDIC
}

\author{
J. Yang ${ }^{1}$, F. Castelli ${ }^{2}$, and Y. Chen ${ }^{1}$ \\ ${ }^{1}$ State Key Laboratory of Desert and Oasis Ecology, Xinjiang Institute of Ecology and Geography, \\ Chinese Academy of Sciences, Xinjiang, 830011, China \\ ${ }^{2}$ Department of Civil and Environmental Engineering, University of Florence, Italy \\ Correspondence to: J. Yang (yangjing@ms.xjb.ac.cn)
}

Received: 28 February 2014 - Published in Hydrol. Earth Syst. Sci. Discuss.: 26 March 2014

Revised: 27 August 2014 - Accepted: 8 September 2014 - Published: 15 October 2014

\begin{abstract}
Calibration of distributed hydrologic models usually involves how to deal with the large number of distributed parameters and optimization problems with multiple but often conflicting objectives that arise in a natural fashion. This study presents a multiobjective sensitivity and optimization approach to handle these problems for the MOBIDIC (MOdello di Bilancio Idrologico DIstribuito e Continuo) distributed hydrologic model, which combines two sensitivity analysis techniques (the Morris method and the state-dependent parameter (SDP) method) with multiobjective optimization (MOO) approach $\varepsilon$-NSGAII (Nondominated Sorting Genetic Algorithm-II). This approach was implemented to calibrate MOBIDIC with its application to the Davidson watershed, North Carolina, with three objective functions, i.e., the standardized root mean square error (SRMSE) of logarithmic transformed discharge, the water balance index, and the mean absolute error of the logarithmic transformed flow duration curve, and its results were compared with those of a single objective optimization (SOO) with the traditional Nelder-Mead simplex algorithm used in MOBIDIC by taking the objective function as the Euclidean norm of these three objectives. Results show that (1) the two sensitivity analysis techniques are effective and efficient for determining the sensitive processes and insensitive parameters: surface runoff and evaporation are very sensitive processes to all three objective functions, while groundwater recession and soil hydraulic conductivity are not sensitive and were excluded in the optimization. (2) Both MOO and SOO lead to acceptable simulations; e.g., for MOO, the average Nash-Sutcliffe value is 0.75 in the calibration period and 0.70 in the validation period. (3) Evaporation and surface
\end{abstract}

runoff show similar importance for watershed water balance, while the contribution of baseflow can be ignored. (4) Compared to SOO, which was dependent on the initial starting location, MOO provides more insight into parameter sensitivity and the conflicting characteristics of these objective functions. Multiobjective sensitivity analysis and optimization provide an alternative way for future MOBIDIC modeling.

\section{Introduction}

With the development of information technology (e.g., highperformance computing cluster and remote sensing technology), there has been a prolific development of integrated, distributed and physically based watershed models (e.g., MIKE-SHE, Refsgaard and Storm, 1995) over the past two decades, which are increasingly being used to support decisions about alternative management strategies in the areas of land use change, climate change, water allocation, and pollution control. Though, in principle, parameters of distributed and physically based models should be assessable from catchment data (in traditional conceptual rainfallrunoff models, parameters are obtained through a calibration process), these models still need a parameter calibration process in practice due to scaling problems, experimental constraints, etc. (Beven and Binley, 1992; Gupta et al., 1998; Madsen, 2003). Problems arising from calibrating distributed hydrologic models include how to handle a large number of distributed parameters and optimization problems with multiple but often conflicting objectives. 
In the literature, to deal with the large number of distributed model parameters, this is often done by aggregating distributed parameters (e.g., Yang et al., 2007) or by screening out the unimportant parameters through a sensitivity analysis (e.g., Muleta and Nicklow, 2005; Yang, 2011). Sensitivity analysis can be used not only to screen out the most insensitive parameters, but also to study the system behaviors identified by parameters and their interactions, qualitatively or quantitatively. However, most applications in environmental modeling are based on a one-at-a-time (OAT) local sensitivity analysis, which is "predicated on assumptions of model linearity which appear unjustified in the cases reviewed" (Saltelli and Annoni, 2010), or simple linear regressions, where a lot of uncertainties are not fairly accounted for. The use of global sensitivity analysis techniques is very crucial in distributed modeling. Only recently, global sensitivity analysis techniques and multiobjective sensitivity analysis started to appear in hydrologic modeling, and van Werkhoven et al. (2009) demonstrates how the calibration result responds to reduced parameter sets with different objectives and different metrics of parameter exclusion.

Although most hydrologic applications are based on the single objective calibration, model calibration with multiple and often conflicting objectives arises in a natural fashion in hydrologic modeling. This is not only due to the increasing availability of multivariable (e.g., flow, groundwater level, etc.) or multisite measurements, but also due to the intrinsic different system responses (e.g., peaks and baseflow in the flow series). Instead of finding a single optimal solution in the single objective optimization (SOO), the task in the multiobjective optimization (MOO) is to identify a set of optimal trade-off solutions (called a Pareto set) between conflicting objectives. Although there are criticisms of MOO, such as that only one parameter set can be used for decision making, recent studies (e.g., Kollat and Reed, 2007) have started to provide the answers. In hydrology, the traditional method to solve multiobjective problems is to form a single objective, e.g., by giving different weights to these multiple objectives or by applying some transfer function. Over the past decade, several MOO algorithm approaches have been applied to the conceptual rainfall-runoff models (e.g., Yapo et al., 1998; Gupta et al., 1998, Madsen, 2000, Boyle et al., 2000; Vrugt et al., 2003; Liu and Sun, 2010), and are now increasingly applied to distributed hydrologic models (e.g., Madsen, 2003; Bekele and Nicklow, 2007; Shafii and Smedt, 2009; MacLean et al., 2010). There are also some papers (Tang et al., 2006; Wöhling et al., 2008) to study their strengths comparatively with their application in hydrology. A good review of MOO applications in hydrological modeling is given by Efstratiadis and Koutsoyiannis (2010). It is worth noting that the multiobjective calibration is different from statistical uncertainty analysis, which is based on the concept (or similar concept) of equifinality (see the discussions in Gupta et al., 1998, and Boyle et al., 2000).

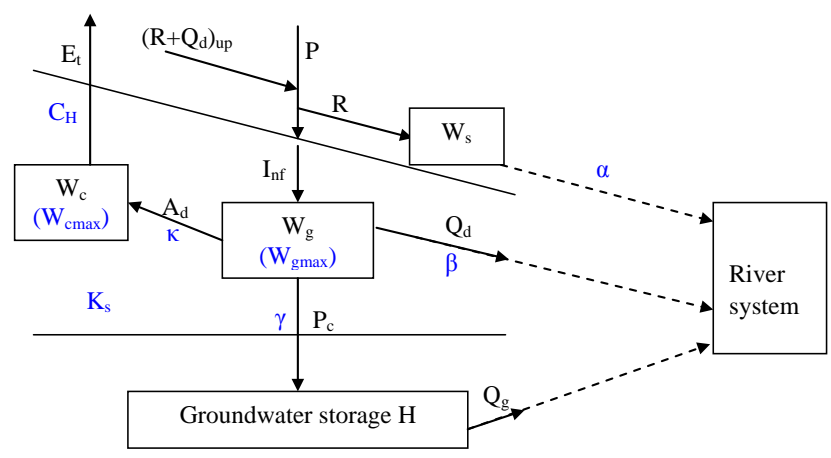

Figure 1. A schematic representation of MOBIDIC. Boxes denote different water storages (gravitational storage $W_{\mathrm{g}}$, capillary storage $W_{\mathrm{c}}$, groundwater storage $H$, surface storage $W_{\mathrm{s}}$, and the river system), solid arrows fluxes (evaporation $E_{\mathrm{t}}$, precipitation $P$, infiltration $I_{\mathrm{nf}}$, adsorption $A_{\mathrm{d}}$, percolation $P_{\mathrm{c}}$, surface runoff $R$, interflow $Q_{\mathrm{d}}$, groundwater discharge $Q_{\mathrm{g}}$, and surface runoff and interflow from upper cells $\left.\left(R+Q_{\mathrm{d}}\right)_{\mathrm{up}}\right)$, dashed arrows different routings, and blue characters major model parameters.

This paper applies two sensitive analysis techniques (the Morris method and the state-dependent parameter (SDP) method) and $\varepsilon$-NSGAII (Non-dominated Sorting Genetic Algorithm-II) in the multiobjective sensitive analysis and calibration framework. This was implemented to calibrate the MOBIDIC (MOdello di Bilancio Idrologico DIstribuito e Continuo) distributed hydrological model with its application to the Davidson watershed, North Carolina. The purpose is to study the parameter sensitivity of the MOBIDIC hydrologic model and to explore the capability of MOO in calibrating the MOBIDIC model compared to the traditional SOO used in MOBIDIC applications.

This paper is structured as follows: Sect. 2 gives a description of the MOBIDIC model; Sect. 3 introduces the approach in the multiobjective sensitivity analysis and optimization; Sect. 4 gives a brief introduction of the study site, the model setup, objective selection, and the sensitivity and calibration procedures; in Sect. 5, the results are presented and discussed; and finally, the main results are summarized and conclusions are drawn in Sect. 6.

\section{MOBIDIC hydrologic model}

MOBIDIC (MOdello di Bilancio Idrologico DIstribuito e Continuo; Castelli et al., 2009; Campo et al., 2006) is a distributed and raster-based hydrological balance model. MOBIDIC simulates the energy and water balances on a cell basis within the watershed. Figure 1 gives a schematic representation of MOBIDIC. The energy balance is approached by solving the heat diffusion equations in multiple layers in the soil-vegetation system, while the water balance is simulated in a series of reservoirs (i.e., the boxes in Fig. 1) and fluxes between them. 
For each cell, water in the soil is simulated by

$$
\begin{aligned}
& \frac{\mathrm{d} W_{\mathrm{g}}}{\mathrm{d} t}=I_{\mathrm{nf}}-S_{\mathrm{per}}-Q_{\mathrm{d}}-S_{\mathrm{as}} \\
& \frac{\mathrm{d} W_{\mathrm{c}}}{\mathrm{d} t}=S_{\mathrm{as}}-E_{\mathrm{t}},
\end{aligned}
$$

where $W_{\mathrm{g}}(\mathrm{L})$ and $W_{\mathrm{c}}(\mathrm{L})$ are the water contents in the soil gravitational storage and capillary storage, respectively, and $I_{\mathrm{nf}}\left(\mathrm{LT}^{-1}\right), S_{\mathrm{per}}\left(\mathrm{LT}^{-1}\right), Q_{\mathrm{d}}\left(\mathrm{LT}^{-1}\right), E_{\mathrm{t}}\left(\mathrm{LT}^{-1}\right)$ and $S_{\mathrm{as}}$ $\left(\mathrm{LT}^{-1}\right)$ are infiltration, percolation, interflow, evaporation, and adsorption from gravitational to capillary storage, which are modeled through the following equations:

$$
\begin{aligned}
S_{\mathrm{per}} & =\gamma \cdot W_{\mathrm{g}} \\
Q_{\mathrm{d}} & =\beta \cdot W_{\mathrm{g}} \\
S_{\mathrm{as}} & =\kappa \cdot\left(1-W_{\mathrm{c}} / W_{\mathrm{c} \max }\right),
\end{aligned}
$$

$I_{\mathrm{nf}}= \begin{cases}{\left[P+\left(Q_{\mathrm{d}}+Q_{\mathrm{h}}+R_{\mathrm{d}}\right)_{\mathrm{up}}\right]} & \\ {\left[1-\exp \left(\frac{-K_{\mathrm{s}}}{P+\left(Q_{\mathrm{d}}+Q_{\mathrm{h}}+R_{\mathrm{d}}\right)_{\mathrm{up}}}\right)\right]} & \text { if } W_{\mathrm{g}}<W_{\mathrm{gmax}} \\ 0 & \text { otherwise }\end{cases}$

where $\gamma, \beta$ and $\kappa$ are the percolation coefficient $\left(\mathrm{T}^{-1}\right)$, the interflow coefficient $\left(\mathrm{T}^{-1}\right)$, and the soil adsorption coefficient (LT ${ }^{-1}$ ), respectively, $P$ the precipitation $\left(\mathrm{LT}^{-1}\right), Q_{\mathrm{h}}$ and $R_{\mathrm{d}}$ the Horton runoff and the Dunne runoff, $K_{\mathrm{S}}$ the soil hydraulic conductivity $\left(\mathrm{LT}^{-1}\right)$, and $W_{\text {gmax }}(\mathrm{L})$ and $W_{\text {cmax }}(\mathrm{L})$ the gravitational and capillary storage capacities.

Once the surface runoff $\left(Q_{\mathrm{h}}\right.$ and $\left.R_{\mathrm{d}}\right)$ and baseflow are calculated, three different methods can be used for river routing, i.e., the lag method, the linear reservoir method, and the Muskingum-Cunge method (Cunge, 1969). The Muskingum-Cunge method was used in this study.

MOBIDIC uses either a linear reservoir or the Dupuit approximation to simulate the groundwater balance, which relates the groundwater change to the percolation, water loss in aquifers and baseflow. In this case study, the linear reservoir method was used.

Although there are many distributed parameters in MOBIDIC, these distributed parameters are normally calibrated through the "aggregate" factors (e.g., the multiplier for hydraulic conductivity) based on their initial estimations, and hereafter we use the term "factor" (instead of "model parameter") when we conduct the sensitivity analysis and optimization, to avoid confusion with the term "model parameter" used in the model description. A factor can be a model parameter or a group of distributed model parameters with the same parameter name, and in this paper, it is a change to be applied to a group of model parameters. In MOBIDIC, nine factors (i.e., nine groups of parameters) normally need to be calibrated. These factors, their explanations, and their corresponding model parameters are listed in Table 1.

\section{Methodology}

The procedure applied here consists of two-step analyses, i.e., a multiobjective sensitivity analysis generally characterizing the basic hydrologic processes and singling out the most insensitive factors, and a multiobjective calibration aiming at trade-offs between different objective functions.

\subsection{Sensitivity analysis techniques}

Sensitivity analysis assesses how variations in model output can be apportioned, qualitatively or quantitatively, to different sources of variations, and how the given model depends upon the information fed into it (Saltelli et al., 2008). In the literature, a lot of sensitivity analysis methods are introduced and applied; e.g., Yang (2011) applied and compared five different sensitivity analysis methods. Here, we adopted an approach that combines two global sensitivity analysis techniques, i.e., the Morris method (Morris, 1991) and the SDP method (Ratto et al., 2007).

\subsubsection{Morris method}

The Morris method is based on a replicated and randomized one-factor-at-a-time design (Morris, 1991). For each factor $X_{i}$, the Morris method uses two statistics, $\mu_{i}$ and $\sigma_{i}$, which measure the degree of factor sensitivity and the degree of nonlinearity or factor interaction, respectively. The higher $\mu_{i}$ is, the more important the factor $X_{i}$ is to the model output, and the higher $\sigma_{i}$ is, the more nonlinear the factor $X_{i}$ is to the model output or more interactions with other factors (for details, refer to Morris, 1991, and Campolongo et al., 2007). The Morris method takes $m *(n+1)$ model runs to estimate these two sensitivity indices for each of $n$ factors with sample size $m$. The advantage is that it is efficient and effective for screening out insensitive factors. Normally $m$ takes values around 50, and according to Saltelli et al. (2008), the sensitivity measure $\left(\mu_{i}\right)$ is a good proxy for the total effect (i.e., $S_{\mathrm{T} i}$ in Eq. 4 below), which is a robust measure in sensitivity analysis.

\subsubsection{State-dependent parameter (SDP) method}

SDP (Ratto et al., 2007) is based on the ANOVA (ANalysis Of VAriance) functional decomposition, which apportions the model output uncertainty (100\%, as 1 in Eq. 3) to factors and different levels of their interactions:

$1=\sum_{i} S_{i}+\sum_{i} \sum_{j>i} S_{i j}+\ldots+S_{12 . . n}$

where $S_{i}$ is the main effect of factor $X_{i}$ representing the average output variance reduction that can be achieved when $X_{i}$ is fixed, and $S_{i j}$ is the first-order interaction between $X_{i}$ and $X_{j}$, and so on. In ANOVA-based sensitivity analysis, the total effect $\left(S_{\mathrm{T} i}\right)$ is frequently used, which stands for the average output variance that would remain as long as $X_{i}$ stays 
Table 1. Initial selected factors, initial estimation of the corresponding MOBIDIC parameter, and factor ranges.

\begin{tabular}{llll}
\hline Factor & $\begin{array}{l}\text { Meaning of the } \\
\text { given factor }\end{array}$ & $\begin{array}{l}\text { Initial estimation of } \\
\text { MOBIDIC parameter }\end{array}$ & $\begin{array}{l}\text { Factor } \\
\text { range }\end{array}$ \\
\hline$p \gamma$ & Exponential change $^{(1)}$ in the soil percolation coefficient $\gamma\left(\mathrm{s}^{-1}\right)$ & $1.2 \times 10^{-11}$ & {$[-2,9]$} \\
$p \kappa$ & Exponential change in the soil adsorption coefficient $\kappa\left(\mathrm{s}^{-1}\right)$ & $1.6 \times 10^{-7}$ & {$[-6,5]$} \\
$p \beta$ & Exponential change in the interflow coefficient $\beta\left(\mathrm{s}^{-1}\right)$ & $2.5 \times 10^{-6}$ & {$[-7,4]$} \\
$p \alpha$ & Exponential change in the surface storage decay coefficient $\alpha\left(\mathrm{s}^{-1}\right)$ & $3.3 \times 10^{-7}$ & {$[-6,5]$} \\
$r K_{\mathrm{S}}$ & Multiplying change ${ }^{(2)}$ in soil hydraulic conductivity $\left(\mathrm{m} \mathrm{s}^{-1}\right)$ & {$\left[5.0 \times 10^{-6}, 8.9 \times 10^{-5}\right]$} & {$[0.001,100]$} \\
$r W_{\mathrm{cmax}}$ & Multiplying change in the maximum storage of the capillary reservoir $(\mathrm{m})$ & {$[0.017,0.165]$} & {$[0.01,5]$} \\
$r W_{\mathrm{gmax}}$ & Multiplying change in the maximum storage of the gravitational reservoir $(\mathrm{m})$ & {$[0.107,0.449]$} & {$[0.01,5]$} \\
$r C_{\mathrm{H}}$ & Multiplying change in the bulk turbulent exchange coefficient for heat $(-)$ & {$[0.010,0.018]$} & {$[0.01,5]$} \\
$r K_{\mathrm{f}}$ & Multiplying change in the groundwater decay coefficient $\left(\mathrm{s}^{-1}\right)$ & $1.0 \times 10^{-7}$ & {$[0.001,5]$} \\
\hline
\end{tabular}

(1) Exponential change $p X$ means the corresponding MOBIDIC parameter $X$ will be changed according to $X=X_{0} \times \exp (p X-1)$, where $X_{0}$ is the initial estimation of $X$.

(2) Multiplying change $r X$ means the corresponding MOBIDIC parameter $X$ will be changed according to $X=X_{0} \times r X$.

unknown.

$S_{\mathrm{T} i}=S_{i}+\sum_{j \neq i} S_{i j}+\ldots+S_{12 \ldots n}$

The SDP method uses the emulation technique to approximate lower-order sensitivity indices in Eq. (3) (e.g., $S_{i}$ and $S_{i j}$ in this study) by ignoring the higher-order sensitivity indices, and we define $S_{D i}=S_{i}+\sum_{j} S_{i j}$ (referred to as the "quasi total effect" later) as a surrogate for the total effect. The advantage is that it can precisely estimate lower-order sensitivity indices at a lower computational cost (normally 500 model runs, which is independent of the number of factors). The disadvantage is that it cannot estimate higher-order sensitivity indices.

In practice, especially for over-parameterized cases, the Morris method is first suggested to screen out insensitive factors, and then the SDP method is applied to quantify the contributions of the sensitive factors and their interactions. In this study, as model parameters are aggregated into nine factors (as listed in Table 1), these two methods are applied individually. Then, the sensitivity of each factor and its system behaviour will be discussed, qualitatively by the Morris method, and quantitatively by the SDP method, and then the most insensitive factors will be screened out and excluded in the calibration.

In the context of multiobjective analysis, the sensitivity analysis applied includes (1) examination of the sensitivity of each factor to different objective functions, qualitatively or quantitatively, (2) singling out of the most sensitive factors and study of the physical behaviors of the system, and (3) exclusion of the most insensitive factors, thereby simplifying the process of calibration. It is worth noting that the sensitivity analysis approach applied here is not a fully multiobjective sensitivity analysis approach such as proposed by Rosolem et al. (2012, 2013), which applies sensitivity analysis to all objectives in an integrated way, and which is objective. However, compared to the fully multiobjctive sensitiv- ity analysis approach (as proposed in Rosolem et al., 2012), which easily requires over 10000 model runs, our approach is very computationally efficient, as both the Morris method and the SDP method only need several hundred model runs, which is highly appreciable for physically based and distributed hydrologic models.

\subsection{Multiobjective calibration and $\varepsilon$-NSGAII}

In the literature of hydrologic modeling, most applications are single objective based, which aims at a single optimal solution. However, for example in flow calibration, there is always a case that for two solutions, one solution simulates the peaks better and simulates the baseflow poorly, while the other solution simulates the peaks poorly and simulates the baseflow better. These two solutions, called Pareto solutions, are incommensurable; i.e., better fitting of the peaks will lead to worse fitting of the baseflow, and vice versa. This belongs to the domain of MOO, aiming at finding a set of optimal solutions (Pareto solutions), instead of one single solution.

Generally, a MOO problem can be formulated as follows:

$$
\begin{aligned}
& \min F(X)=\left(f_{1}(X), f_{2}(X), \ldots, f_{i}(X), \ldots, f_{k}(X)\right) \\
& \text { s.t. } G(X)=\left(g_{1}(X), g_{2}(X), \ldots, g_{i}(X), \ldots, g_{l}(X)\right),
\end{aligned}
$$

where $X$ is an $n$-dimensional vector and, in this study, represents the model factors to be calibrated, $f_{i}(X)$ is the $i$ th objective function, and $g_{i}(X)$ is the $i$ th constraint function.

In the literature, there are many algorithms available to obtain the Pareto solutions, e.g., NSGAII (Deb et al, 2002), SPEA2 (Strength Pareto Evolutionary Algorithm 2; Zitzler et al., 2001), MOSCEM-UA (Multiobjective Shuffled Complex Evolution Metropolis; Vrugt et al., 2003), and $\varepsilon$-NSGAII (Kollat and Reed, 2006), etc. In this study, we adopt $\varepsilon$ NSGAII, which is efficiency, reliability, and ease of use. Its strengths have been comparatively studied in Kollat and Reed (2006) and Tang et al. (2006).

$\varepsilon$-NSGAII is an extension of NSGAII (Deb et al., 2002), a second generation of multiobjective evolution algorithms. 
The main characteristics of $\varepsilon$-NSGAII include the (i) selection, crossover, and mutation processes as with other genetic algorithms by mimicking the process of natural evolution, (ii) an efficient non-domination sorting scheme, (iii) an elitist selection method that greatly aids in capturing Pareto fronts, (iv) $\varepsilon$ dominance archiving, (v) adaptive population sizing, and (vi) automatic termination to minimize the need for extensive parameter calibration. For more details, refer to Kollat and Reed (2006). In this study, two changes were made to the original $\varepsilon$-NSGAII: (1) the initial population is generated with the Sobol quasi-random sampling technique to improve the coverage of parameter space; and (2) the code is parallelized and interfaced with MOBIDIC to improve the computational speed.

As a comparison, a single objective function is defined as the 2-norm of the multiple objectives $F(X)$, which measures how close they are to the original point (theoretical optimum O):

$\operatorname{sof}=\|F(\boldsymbol{X})\|_{2}=\sqrt{\sum_{i=1}^{k} f_{i}(\boldsymbol{X})^{2}}$,

and SOO was done with the classic Nelder-Mead algorithm (Nelder and Mead, 1965), which is already coded into the MOBIDIC package.

To analyze the Pareto solution and also to compare it with the solution from SOO, except for traditional methods, the "level diagram" proposed by Blasco et al. (2008) was also used. Compared to traditional methods, it can visualize highdimensional Pareto fronts, and synchronizes the objective and factor diagrams. The procedure includes two steps. In the first step, the vector of objectives ( $k$ dimension) for each Pareto point is mapped to a real number (one dimension) according to the proximity to the theoretical optimum measured with a specific norm of objectives; and in the second step, these norm values are plotted against the corresponding values of each objective or factor. 1-norm, 2-norm and $\infty$-norm are suggested. For comparison with SOO, 2-norm was used.

\section{Davidson watershed and objective selection}

\subsection{Davidson watershed}

The Davidson watershed, located in the southwestern mountain area of North Carolina, drains an area of $105 \mathrm{~km}^{2}$ above the Davidson River station near Brevard (see Fig. 2). The elevation ranges from 645 to $1820 \mathrm{~m}$ above sea level. Based on the North American Land Data Assimilation System (NLDAS) climate data, the average annual precipitation is $1900 \mathrm{~mm}$, and varies from $1400 \mathrm{~mm}$ to $2500 \mathrm{~mm}$, and daily temperature changes from -19 to $26^{\circ} \mathrm{C}$. The average daily flow is about $3.68 \mathrm{~m}^{3} \mathrm{~s}^{-1}$.

Data used in the MOBIDIC model include (i) a digital elevation model (DEM), (ii) soil data, (iii) land cover

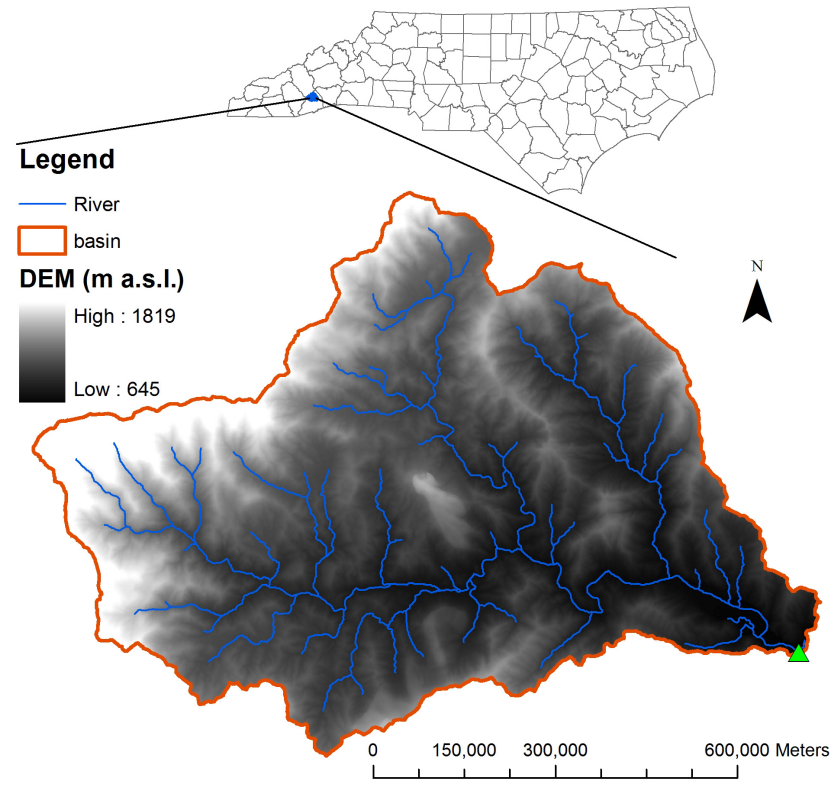

Figure 2. The location of the Davidson watershed, North Carolina, with a DEM map, the river system (lines), and the watershed outlet (the triangle point).

data, (iv) climate data (precipitation, minimum and maximum temperature, solar radiation, humidity and wind speed) and (v) flow data; a DEM of $9 \mathrm{~m}$, land cover, SSURGO soil data, one station (the Davidson River near Brevard) of flow data from the US Geological Survey, and hourly NLDAS climate data from the National Aeronautics and Space Administration (NASA). NLDAS integrates a large quantity of observation-based and model reanalysis data to drive offline (not coupled to the atmosphere) land-surface models (LSMs), and runs at 1/8 degree grid spacing over central North America, enabled by the Land Information System (LIS) (Kumar et al., 2006; Peters-Lidard et al., 2007).

A DEM is used to delineate the watershed and to estimate the topographic parameters and the river system, land cover for evaporation parameters, and soil data for soil parameters. Climate data are used to drive MOBIDIC, and flow data are used to calibrate the model and to assess model performance. The climate and flow data used in this study are from 1 January 1996 to 30 September 2006. As NLDAS only has hourly temperature daily instead of the hourly minimum and maximum temperatures needed by MOBIDIC, we compiled the hourly climate data to daily data and ran the model at a daily step. After MOBIDIC setup, the initial parameter values are listed in the third column of Table 1.

We split the data into a warm-up period (from 1 January 1996 to 30 September 2000), a calibration period (from 1 October 2000 to 30 September 2003), and a validation period (from 1 October 2003 to 30 September 2006). 


\subsection{Objective function selection}

After setting up MOBIDIC in the Davidson watershed, three objective functions were used in the multiobjective sensitivity analysis and optimization:

1. Standardized root mean square error (SRMSE) between the logarithms of simulated and observed outflows:

$$
\text { SRMSE }=\frac{\sqrt{\frac{1}{N} \sum_{i=1}^{N}\left(\log \left(Q_{i}^{\text {obs }}\right)-\log \left(Q_{i}^{\text {sim }}\right)\right)^{2}}}{\sqrt{\frac{1}{N-1} \sum_{i=1}^{N}\left(\log \left(Q_{i}^{\text {obs }}\right)-\log Q\right)^{2}}}
$$

2. Water balance index (WBI), calculated as the mean absolute error between the simulated and observed flow accumulation curves:

$$
\mathrm{WBI}=\frac{1}{N} \sum_{i=1}^{N}\left|Q_{\mathrm{C} i}^{\mathrm{obs}}-Q_{\mathrm{C} i}^{\mathrm{sim}}\right|
$$

3. Mean absolute error between the logarithms of simulated and observed flow duration curves:

$$
\operatorname{MARD}=\frac{1}{100} \sum_{i=1}^{N}\left|\log \left(Q_{\mathrm{P} i}^{\mathrm{obs}}\right)-\log \left(Q_{\mathrm{P} i}^{\mathrm{sim}}\right)\right|
$$

In Eqs. (7), (8) and (9), $Q_{i}^{\text {obs }}$ and $Q_{i}^{\text {sim }}$ are observed and simulated flow series at time step $i, N$ the data length, $\overline{\log Q}$ the average of logarithmic transformed observed flows, $Q_{\mathrm{C} i}^{\mathrm{obs}}$ and $Q_{\mathrm{C} i}^{\text {sim }}$ the $i$ th observed and simulated accumulated flows, and $Q_{\mathrm{P} i}^{\mathrm{obs}}$ and $Q_{\mathrm{P} i}^{\mathrm{sim}}$ the $i$ th percentiles of observed and simulated flow duration curves.

SRMSE (Eq. 7), WBI (Eq. 8) and MARD (Eq. 9) are measures of the closeness between simulated and observed flow series, water balance, and the closeness between simulated and observed flow frequencies, respectively. The smaller these measures are, the better the simulation is, and the minima are $(0,0,0)$, meaning a perfect match between the simulation and the observation. It is worth noting that we use the logarithms of the flows instead of flows to avoid overfitting flow peaks (Boyle et al., 2000; Shafii and De Smedt, 2009), as flood forecasting is not our main focus, and for SRMSE, we have NS approximately equal to $1 \mathrm{SRMSE}^{2}$ when $N$ is large (e.g., > 100), where NS is the Nash-Sutcliffe coefficient (Nash and Sutcliffe, 1970), which is widely used in hydrologic modeling.

Accordingly, the single objective function here is the Euclidean norm (2-norm) of SRMSE, WBI and MARD:

sof $=\sqrt{\mathrm{SRMSE}^{2}+\mathrm{WBI}^{2}+\mathrm{MARD}^{2}}$

\section{Result and discussion}

\subsection{Multiobjective sensitivity analysis}

The Morris method and the SDP method were applied individually to the initially selected factors (in Table 1).
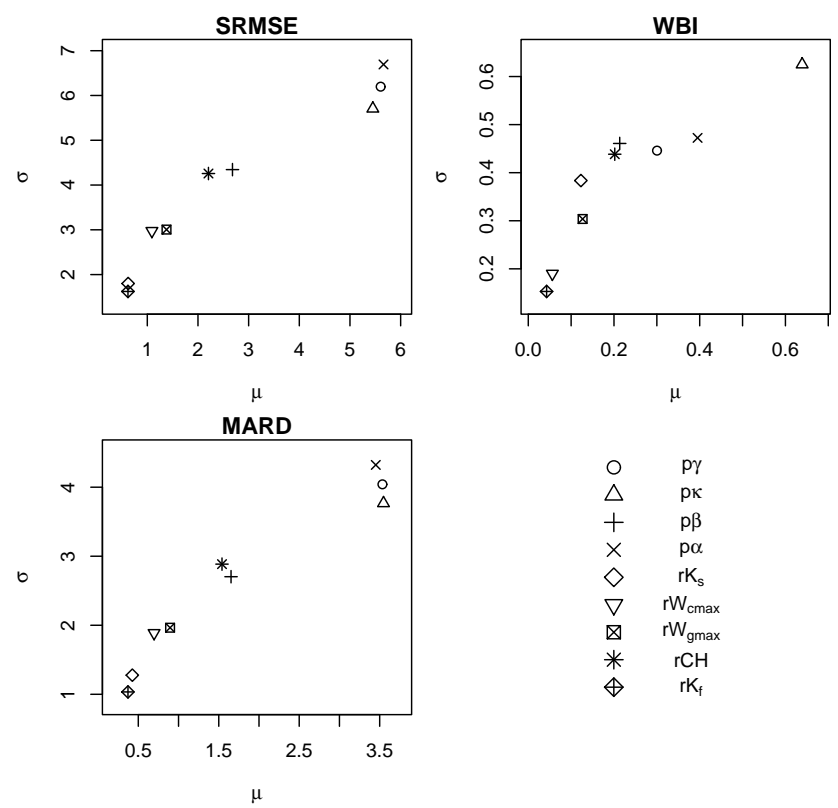

Figure 3. Multiobjective sensitivity analysis result based on the Morris method ( $\mu$ is the sensitivity measure, and $\sigma$ demonstrates the degree of nonlinearity or factor interaction).

For the Morris method, its convergences for three objective functions, monitored using the method proposed in Yang (2011), were achieved with around $700 \sim 800$ model simulations. Figure 3 gives the sensitivity results for objective functions SRMSE, WBI, and MARD, respectively. In each plot, the horizontal axis $(\mu)$ denotes the degree of factor sensitivity, and the vertical axis $(\sigma)$ denotes the degree of factor nonlinearity or interaction with other factors.

For SRMSE, the most sensitive factors are group ( $p \alpha, p \gamma$, and $p \kappa$ ), followed by $p \beta$ and $r C H$, while other factors (especially $r K_{\mathrm{S}}$ and $r K_{\mathrm{f}}$ ) are not so sensitive. This applies to the degree of the factor nonlinearity or interaction. Factors in the same group have a similar effect on the studied objective function. The sensitivities of $p \alpha, p \gamma$, and $p \kappa$ indicate the importance of their corresponding processes (i.e., surface runoff, percolation, and adsorption, which is related to evapotranspiration) for SRMSE, while interflow $(p \beta)$ is less important, and other processes/characteristics (e.g., groundwater flow and $r K_{\mathrm{f}}$ ) are not important.

For WBI, the dominating parameter is $p \kappa$, followed by $p \alpha, p \gamma, p \beta$ and $r C H$, while other factors (especially $r K_{\mathrm{f}}$ and $\left.r W_{\text {cmax }}\right)$ are not so sensitive. WBI measures the water balance between observed and simulated flow series, and it is reasonable that $p \kappa$, which controls the water supply for evaporation, is most sensitive, while other factors $(p \alpha, p \gamma$, $p \beta$ and $r \mathrm{CH}$ ) are sensitive mainly through interaction with this factor, as indicated by the high $\sigma$ of these factors.

For MARD, the results are nearly the same as SRMSE, and this means factors behave similarly to these two objective functions. 


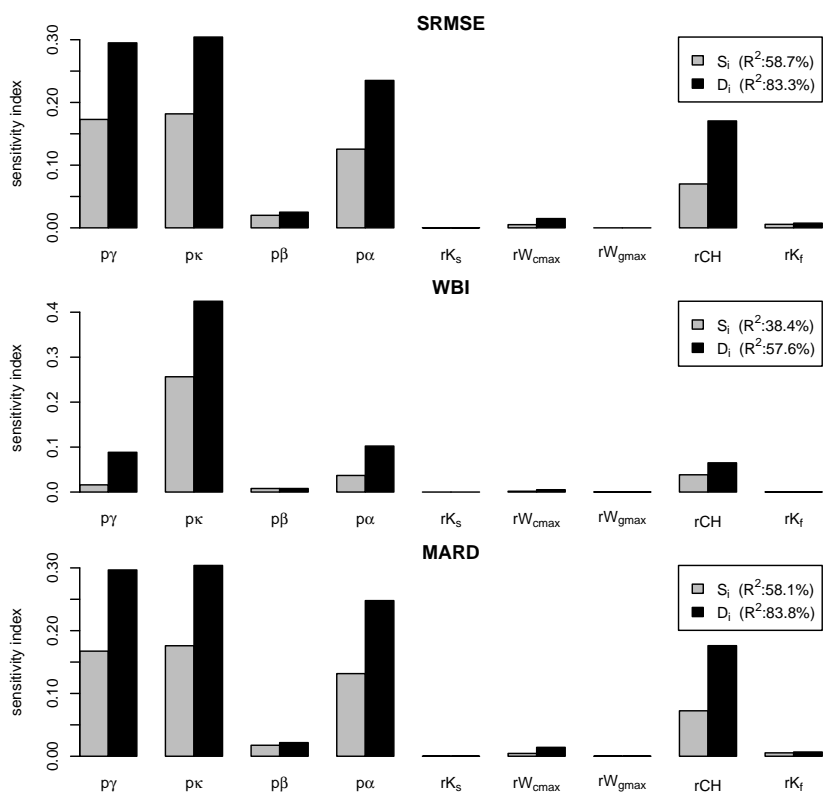

Figure 4. Multiobjective sensitivity analysis result based on the SDP method.

Figure 4 gives the sensitivity results based on the SDP method for SRMSE, WBI, and MARD, from top to bottom. In each plot, the grey and black bars are $S_{i}$ and $S_{\mathrm{D} i}$ for each factor.

For SRMSE, as indicated by $R^{2}$ in the legend, the main effects $\left(S_{i}\right)$ contribute up to $58.7 \%$ of the SRMSE uncertainty, and quasi total effects $\left(S_{\mathrm{D} i}\right)$ account for $83 \%$ of the SRMSE uncertainty, which is quite high, while another $17 \%$ due to higher interactions are not explained. Based on $S_{\mathrm{D} i}$ (black bar), the most sensitive factors are $p \gamma$ and $p \kappa$, followed by $p \alpha$ and $r C H$, and then $p \beta$ and $r W_{\mathrm{cmax}}$, while other factors are not sensitive. This result quantitatively corroborates the result obtained from the Morris method. The main effects $\left(S_{i}\right)$ of $p \gamma, p \kappa$ and $p \alpha$ are high (i.e., $0.17,0.18$ and 0.14 ), which suggests that these factors should be determined first in model calibration, as they lead to the largest reduction in SRMSE uncertainty. For each factor, the difference between the black bar and the grey bar shows the first-order interaction with other factors. This interaction is very strong in $p \gamma$, $p \kappa, p \alpha$ and $r C H$, and is very weak in other factors.

For WBI, as indicated by $R^{2}$ in the legend, the total main effects $\left(S_{i}\right)$ contribute up to $38.4 \%$ of the WBI uncertainty, quasi total effects $\left(S_{\mathrm{D} i}\right)$ only account for $57.6 \%$ of the WBI uncertainty, and around $40 \%$ due to higher interactions are not explained and can not be ignored. However, by comparing the result with that from the Morris method (top-right corner in Fig. 3), we still can get some valuable results: the dominating sensitive factor is $p \kappa$, with $S_{\mathrm{D} i}$ equal to 0.43 (which is the same as the Morris method), followed by $p \gamma$, $p \alpha$ and $r C H$, while other factors are not sensitive; the main effect of $p \kappa$ is as high as 0.27 , and it should be fixed in order

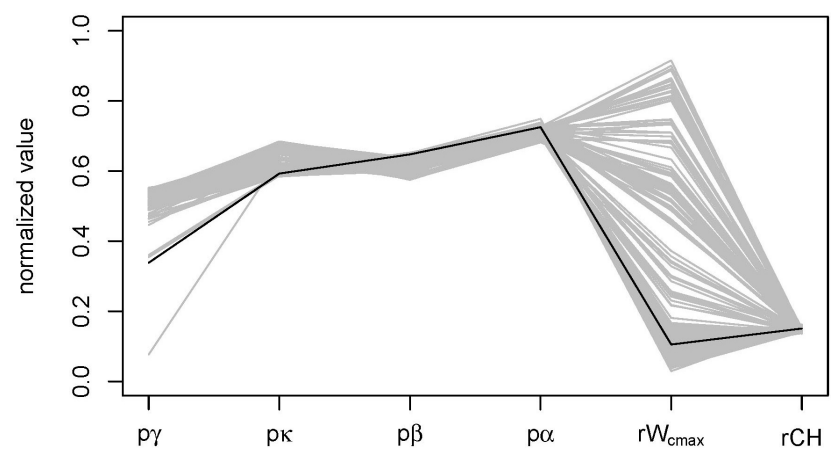

Figure 5. The normalized factor sets associated with MOO (grey lines) and the solution with SOO (dark line).

to get the maximum reduction in WBI uncertainty; the first interaction is high in $p \kappa, p \gamma$ and $p \alpha$, and is not obvious in other factors.

Similar to the Morris model results for SRMSE and MARD, the result of MARD is nearly the same as SRMSE. The similar result for SRMSE and MARD shows a similar characteristic relationship between the factors and the objective function. This is explainable: a good simulation measured by SRMSE will more likely result in a good measure of MARD, and vice versa.

As aforementioned, in the context of multiobjective sensitivity analysis, sensitivity analysis excludes factors that are insensitive to all the objective functions considered. Based on the analysis above, the four most insensitive factors are $r K_{\mathrm{s}}, r K_{\mathrm{f}}, r W_{\mathrm{cmax}}$ and $r W_{\mathrm{gmax}}$. However, as shown in Fig. 4 , $r W_{\text {cmax }}$ is more sensitive than the other three factors, and for the objective function WBI, as higher-order interactions are strongly based on SDP (i.e., explaining around $40 \%$ of model uncertainty), evaporation is the most sensitive process to water balance (as indicated by $p \kappa$ and $r C H$ ), and $r W_{\text {cmax }}$ is the only factor related to evaporation storage $\left(W_{\mathrm{c}}\right)$; therefore, we only exclude $r K_{\mathrm{f}}, r K_{\mathrm{s}}$ and $r W_{\mathrm{gmax}}$ for calibration.

\subsection{Multiobjective optimization}

After sensitivity analysis, only six factors were involved in the calibration. For MOO, we set the initial population size to 128 to obtain a good coverage of the factor space and other $\varepsilon$-NSGAII parameters to their recommended values, and it led to 482 Pareto front points from a total of 22000 model runs with modified $\varepsilon$-NSGAII. For SOO, it stopped after 686 model runs with the classic Nelder-Mead algorithm. Apparently, $\varepsilon$-NSGAII took more model simulations than the Nelder-Mead algorithm, but simulation time was compensated for by the parallelized code running on highperformance clusters. 

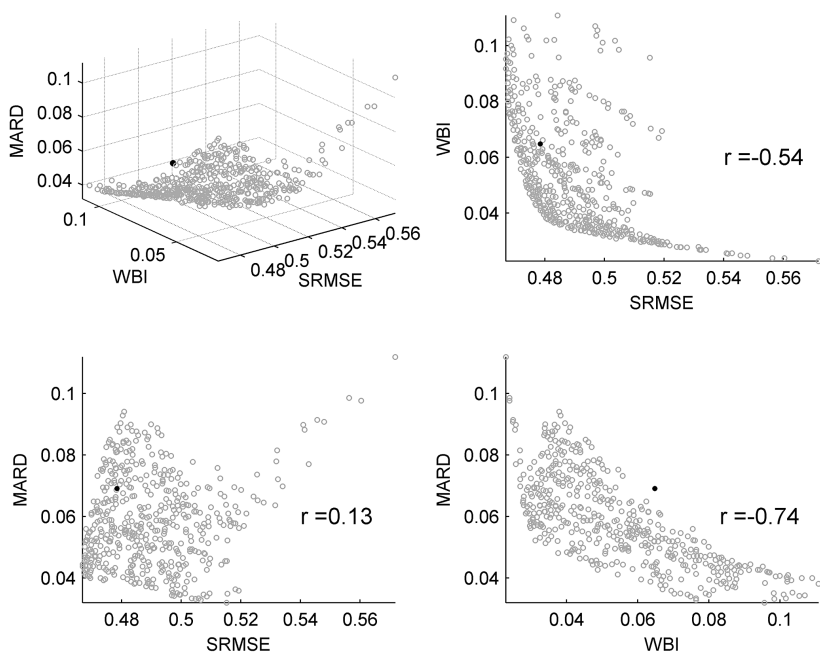

Figure 6. The Pareto solutions in the three-dimensional space (top left), and the projections in the two-dimensional subspace (other plots), with MOO, and the black dot is the solution with SOO.

Figure 5 shows optimized non-dominant sets normalized within $[0,1]$, and the black line is for the factor set with SOO. It is encouraging that, except for $r W_{\text {cmax }}$, factor ranges decreased a lot. This corroborates the conclusion in the sensitivity analysis: $p \gamma, p \kappa, p \beta, p \alpha$, and $r C_{\mathrm{H}}$ are the most sensitive and identifiable factors in these three objective functions, while $r W_{\text {cmax }}$ is less sensitive and less identifiable. Several scattered values of $p \gamma$ and dispersed $r W_{\text {gmax }}$ show that optimized factor sets are scattered in the response surface rather than concentrated in a continuous region, and the factor set with $\mathrm{SOO}$ is within the range of non-dominant sets.

Figure 6 shows Pareto solutions scattered in the threedimensional space (top left), and projections in twodimensional subspaces with the corresponding correlation coefficients $(r)$ in the calibration period, with the black dot in each plot denoting the solution for SOO. Correlation coefficients are high and negative for SRMSE and WBI $(-0.54)$, and for WBI and MARD $(-0.74)$, and this indicates strong trade-off interactions along the Pareto surface; i.e., a better (lower) WBI will eventually result in a worse (higher) SRMSE, and vice versa. The correlation coefficient is low (0.13) between SRMSE and MARD, and is even lower when these two objectives approach their minimum regions (i.e., SRMSE $<0.53$ and MARD < 0.09 ). This might indicate a poor choice of the objective function, as also shown by similar sensitivity results for these two objective functions in Sect. 5.1. Table 2 lists the statistics of these three objectives associated with Pareto sets and the result of SOO. For Pareto sets, in the calibration period, the average SRMSE is 0.49, ranging from 0.47 to 0.57 , which corresponds to the average NS of 0.78 , ranging from 0.67 to 0.78 ; the average WBI is 0.05 , ranging from 0.02 to 0.11 , and the average MEAD is 0.08 , ranging from 0.03 to 0.11 . In the validation period, the average SRMSE is 0.54 , ranging from 0.51 to 0.62 , which corresponds to an average NS of 0.70 , ranging from 0.61 to 0.74; the average WBI is 0.05 , ranging from 0.04 to 0.09 , and the average MEAD is 0.10 , ranging from 0.08 to 0.13 . For SOO, SRMSE, WBI and MEAD are $0.48,0.06$ and 0.07 for the calibration period, and $0.57,0.06$ and 0.10 for the validation, and accordingly the NS values are 0.77 and 0.67 , respectively. According to Moriasi et al. (2007), which suggests NS $>0.75$ and $\mathrm{WBI}<10 \%$ as excellent modeling of river discharge, all Pareto solutions with $\mathrm{MOO}$ and the solution with SOO are close to "excellent" for both the calibration and validation periods.

To visualize Pareto sets better and to compare them with the result of SOO, the level diagrams are plotted in Fig. 7 by applying a Euclidean norm (2-norm) to evaluate the distance of each Pareto point to the ideal origin $(0,0,0)$ (the ideal values for all three normalized objectives are 0). In Fig. 7, the top three plots are for the three objectives, the rest are for optimized factors, and the black dot in each plot is the solution for SOO. In the level diagrams, each objective and each factor of a point (corresponding to a Pareto solution) is represented by the same 2-norm value for all the plots. Compared with MOO, obviously, SOO was trapped in the local optima, as seen in the top-left plot. Another SOO was done with its starting point close to the optimum of MOO, and now the optimum of SOO is very close to that of MOO, which means that optimization with the Nelder-Mead algorithm was dependent on the starting point. The 2-norm has a close linear relationship with SRMSE due to values of SRMSE being 5 to 10 times those of the other two objective functions, and it does not have such a relationship with the other two objectives. The scattering of objectives and factors makes it difficult in decision making to select a single solution, because there is no clear trade-off solution (Blasco et al., 2008). However, compared with SOO, the Pareto solutions from MOO can make decision making easy, as it can be converted with expert opinion or some utility function.

Figures 8 and 9 show simulated and observed flow duration curves and time series flows, respectively, with grey lines denoting the simulations with MOO and black lines with SOO. Generally, all simulations match the observation well for both the duration curve and the time series flow for both calibration period and validation period. For the duration curve, simulations from MOO show a wide range in the low flows, with frequencies from 0.85 to 1.0 , which reflects the insensitivity of groundwater process (discussed in the sensitivity analysis; i.e., $r K_{\mathrm{f}}$ is insensitive to these three objectives). Except for this, there is a slight overestimation of flows: large flows during the calibration period with frequencies from 0.2 to 0.1 , and median to large flows during the validation period with frequencies from 0.5 to 0.1 . This might be due to the uncertainty in the reanalyzed climate data, and the extreme flow with frequencies around 0 is underestimated. This is because we chose the logarithm scale of the observed and simulated flows instead of the normal 
Table 2. Statistics of three objective functions associated with multiobjective optimization and single objective optimization.

\begin{tabular}{|c|c|c|c|c|c|c|c|c|}
\hline & \multicolumn{6}{|c|}{ Multiobjective optimization } & \multicolumn{2}{|c|}{ Single objective optimization } \\
\hline & \multicolumn{3}{|c|}{ Calibration } & \multicolumn{3}{|c|}{ Validation } & \multirow[t]{2}{*}{ Calibration } & \multirow[t]{2}{*}{ Validation } \\
\hline & Mean & Min & Max & Mean & Min & Max & & \\
\hline SRMSE & 0.49 & 0.47 & 0.57 & 0.54 & 0.51 & 0.62 & 0.48 & 0.57 \\
\hline WBI & 0.05 & 0.02 & 0.11 & 0.05 & 0.04 & 0.09 & 0.07 & 0.06 \\
\hline MARD & 0.08 & 0.03 & 0.11 & 0.10 & 0.08 & 0.13 & 0.07 & 0.10 \\
\hline
\end{tabular}
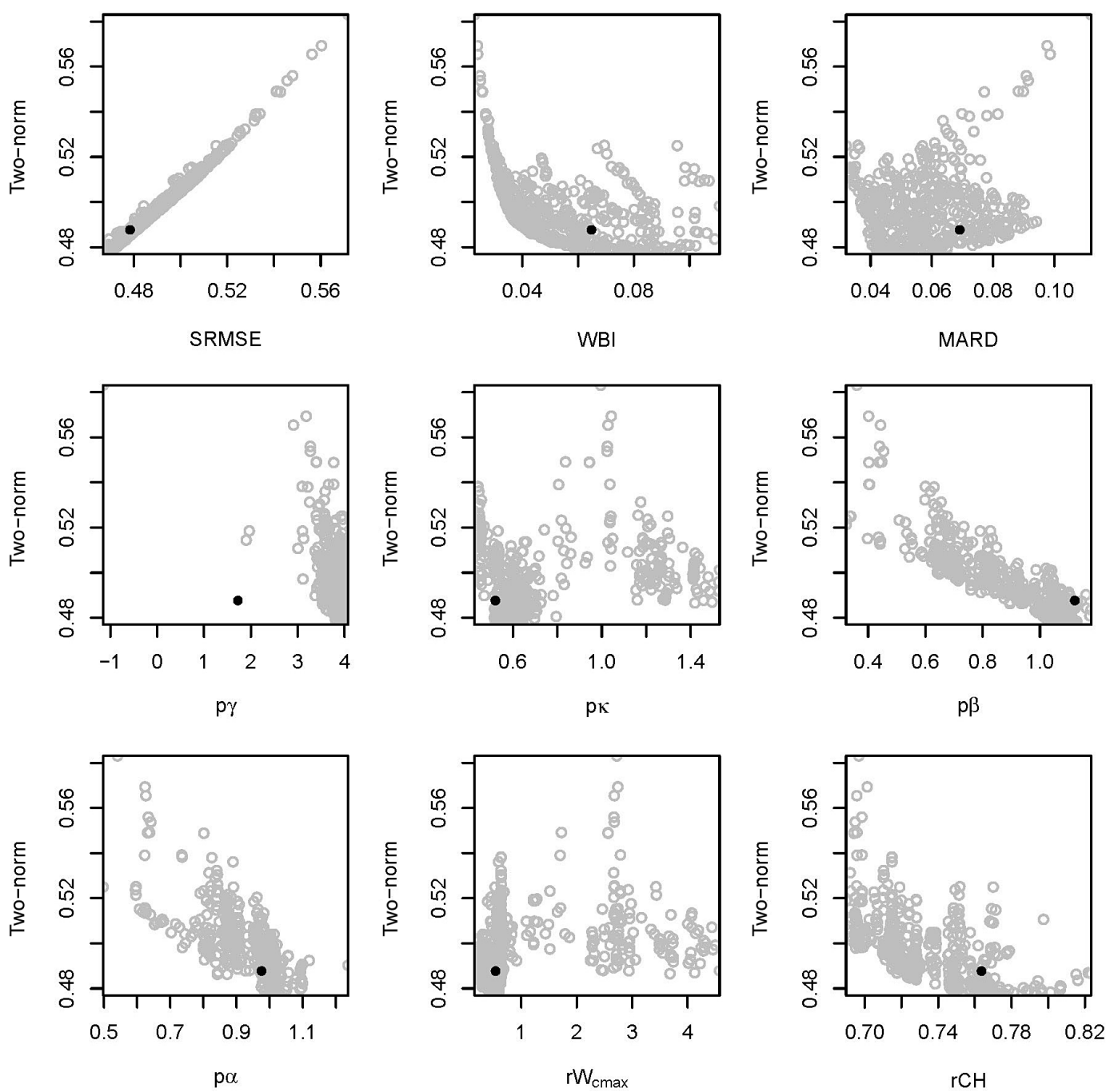

Figure 7. Two-norm level diagram representation of the Pareto sets with MOO, and the solution with SOO (black dot).

scale when computing objectives SRMSE and MARD. With $\mathrm{SOO}$, the deviation from the observation is larger. Similar conclusions can be drawn from the time series simulations in Fig. 9, i.e., the wide ranges of low-flow periods, and underestimation of flow peaks. Other than this, all simulations can generally mimic the observations.
Figure 10 shows the time series of average watershed storage (soil storage expressed as soil saturation, and groundwater depth) and fluxes (evaporation, surface runoff and baseflow) associated with MOO (shaded) and SOO (black line). With MOO, soil saturation varies from 0.2 to 1.0 , and groundwater from 0 to $120 \mathrm{~mm}$. The temporal fluctuation of soil moisture is higher than groundwater, but lower than fluxes in evaporation and surface runoff, and this is true for 

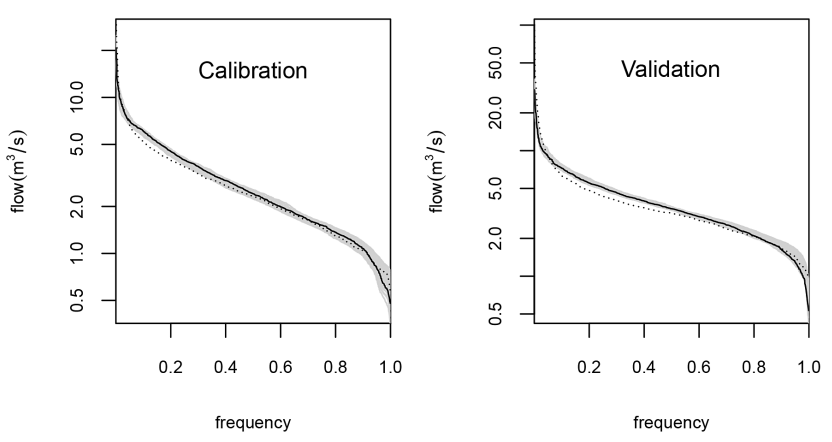

Figure 8. Flow duration curve for observations (dotted line), and simulated with MOO (grey) and SOO (solid line).
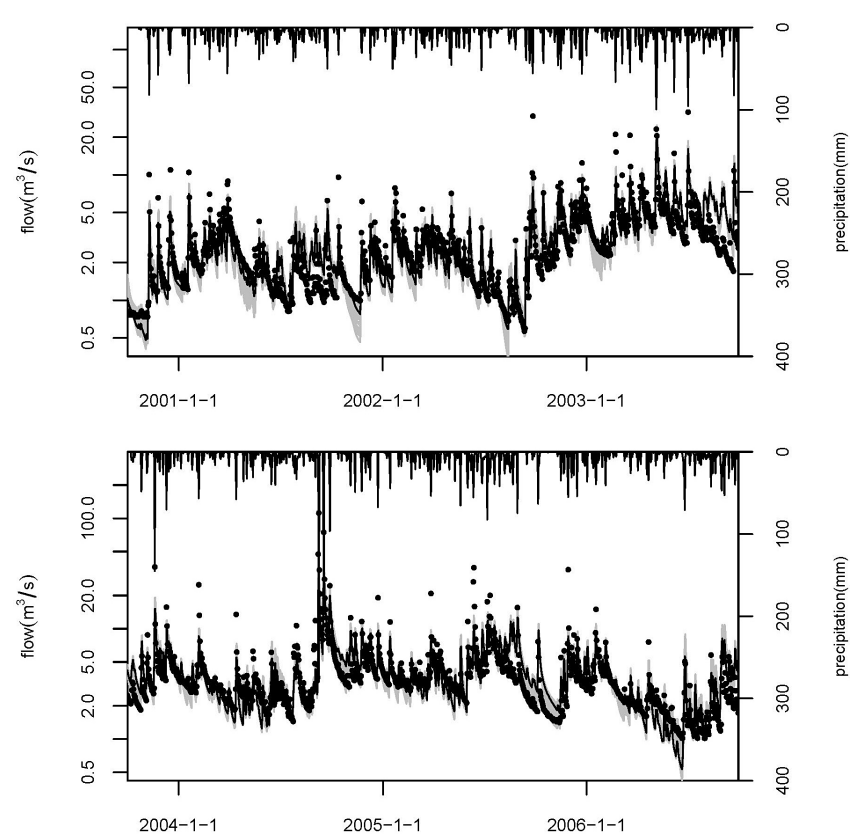

Figure 9. Observed flows (dotted) and simulated flows with MOO (grey) and SOO (black line) for the calibration period (top) and validation period (bottom).

the solution with SOO, except for its ranges of soil saturation and groundwater (groundwater is very close to $0 \mathrm{~mm}$ ). For fluxes with MOO, evaporation and surface runoff have more temporal variation than baseflow, and their magnitudes are larger than baseflow. This applies to fluxes with SOO, and its baseflow is close to 0 . This can be confirmed by the De Finetti diagram in Fig. 11: with MOO, the average contributions of evaporation, surface runoff, and baseflow are 49.3, 46.1 , and $4.8 \%$, respectively, while the contribution of baseflow is very insignificant, and the contribution of baseflow is almost 0 with SOO.
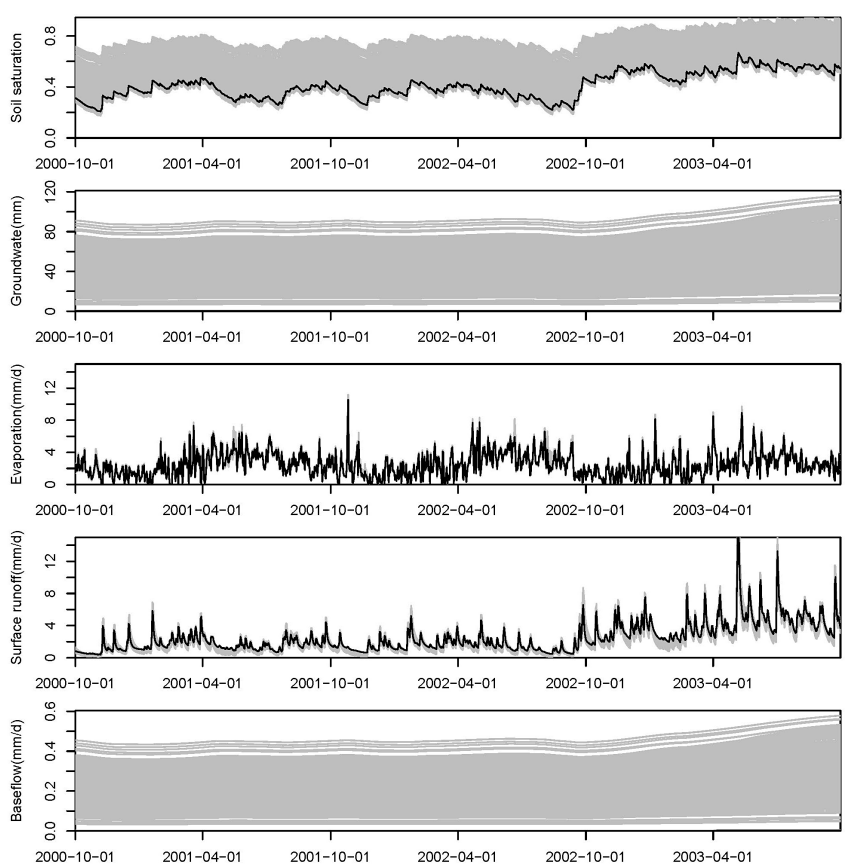

Figure 10. Time series of watershed average storages (soil water storage expressed as soil saturation, and groundwater depth), and fluxes (evaporation, surface runoff, and baseflow) with MOO (grey) and SOO (black line). For SOO, the groundwater storage and baseflow are close to 0 and hardly seen.

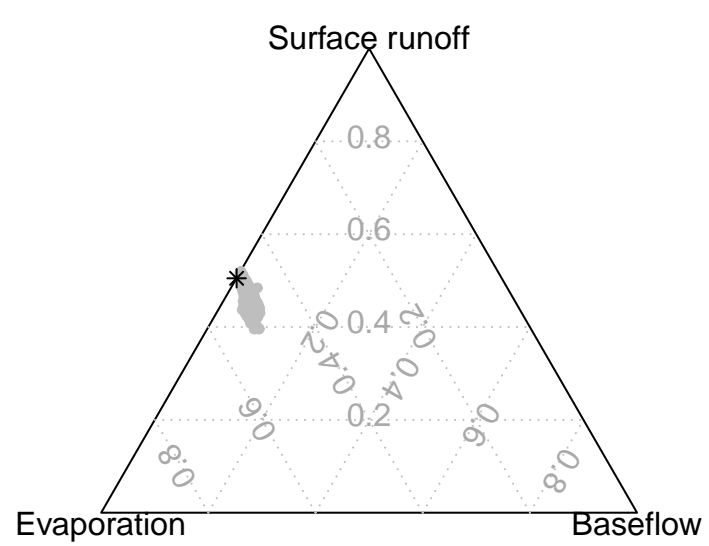

Figure 11. De Finetti diagram (ternary plot) of evaporation, surface runoff, and baseflow with MOO (grey) and SOO (black star).

The result of MOO above is based on a single random seed. The result of MOO with another random seed is similar to the above, except that the range of $r W_{\text {cmax }}$ is narrower (however, its effect on the simulation result is limited due to its low sensitivity that was discussed). Multiple-rand-seed MOO is always appealing, but it might not be practical for fully distributed and physically based models, which are normally time consuming in computation. What one can do is to choose a reliable and robust algorithm based on a literature review. 


\section{Conclusions}

This study presents a multiobjective sensitivity and optimization approach to calibrating the MOBIDIC distributed hydrologic model with its application in the Davidson watershed for three objective functions (i.e., SRMSE, WBI and MARD). Results show that

1. The two sensitivity analysis techniques are effective and efficient in determining the sensitive processes and insensitive parameters: surface runoff and evaporation are very sensitive processes to all three objective functions, while groundwater recession and soil hydraulic conductivity are not sensitive and were excluded from the optimization.

2. For SRMSE and MARD, all the factors have almost the same sensitivities, and a low correlation exists between these two objectives in the non-dominance of the Pareto set. This might indicate a poor choice of the objective function.

3. Both MOO and SOO achieved acceptable results for both the calibration period and the validation period in terms of objective functions and a visual match between simulated and observed flows and flow duration curves. For example, with MOO, the average NS values are 0.75 , ranging from 0.67 to 0.78 in the calibration period, and 0.70 , ranging from 0.61 to 0.74 in the validation period.

4. In the case study, evaporation and surface runoff show similar importance to the watershed water balance, while the contribution of baseflow can be ignored.

5. Comparing MOO with $\varepsilon$-NSGAII, the application of SOO with the Neld-Mead algorithm was dependent on an initial starting point. Furthermore, the Pareto solution provides a better understanding of these conflicting objectives and the relations between objectives and parameters, and a better way for decision making.

Acknowledgements. The research was supported by the Thousand Youth Talents plan (Xinjiang project) and the National Basic Research Program of China (973 Program: 2010CB951003). The data used in this study were acquired as part of the mission of NASA's Earth Science Division and archived and distributed by the Goddard Earth Sciences (GES) Data and Information Services Center (DISC). The authors would like to thank Rafael Rosolem and the other two anonymous reviewers for valuable comments that substantially improved the manuscript.

Edited by: D. Solomatine

\section{References}

Bekele, E. G. and Nicklow, J. W.: Multi-objective automatic calibration of SWAT using NSGA-II, J. Hydrol., 341, 165-176, 2007

Beven, K. and Binley, A.: The future of distributed models - model calibration and uncertainty prediction, Hydrol. Process., 6, 279 298, 1992.

Blasco, X., Herrero, J. M., Sanchis, J., and Martínez, M.: A new graphical visualization of n-dimensional Pareto front for decision-making in multiobjective optimization, Inform. Sciences, 178, 3908-3924, 2008

Boyle, D., Gupta, H., and Sorooshian, S.: Toward improved calibration of hydrologic models: combining the strengths of manual and automatic methods, Water Resour. Res., 36, 3663-3674, 2000.

Campo, L., Caparrini, F., and Castelli, F.: Use of multi-platform, multi-temporal remote-sensing data for calibration of a distributed hydrological model: an application in the Arno basin, Italy, Hydrol. Process., 20, 2693-2712, 2006

Campolongo, F., Cariboni, J., and Saltelli, A.: An effective screening design for sensitivity analysis of large models, Environ. Modell. Softw., 22, 1509-1518, 2007.

Castelli, F., Menduni, G., Caparrini, F., and Mazzanti, B.: A distributed package for sustainable water managment: a case study in the Arno basin, in: The Role of Hydrology in Water Resoures Management (Proceedings of a symposium held on the island of Capri, Italy, October 2008), IAHS Publ. 327, 2009.

Cunge, J. A.: On the subject of a flood propagation method (Muskingum method), J. Hydraul. Res., 7, 205-230, 1969.

Deb, K., Pratap, A., Agarwal, S., and Meyarivan, T.: A fast and elitist multiobjective genetic algorithm: NSGA-II, IEEE T. Evolut. Comput., 6, 182-97, 2002.

Efstratiadis, A. and Koutsoyiannis, D.: One decade of multiobjective calibration approaches in hydrological modelling: a review, Hydrol. Sci. J., 55, 58-78, 2010

Gupta, H., Sorooshian, S., and Yapo, P.: Toward improved calibration of hydrologic models: multiple and noncommensurable measures of information, Water Resour. Res., 34, 751-763, 1998.

Kollat, J. B. and Reed, P. M.: Comparing state-of-the-art evolutionary multiobjective algorithms for long-term groundwater monitoring design, Adv. Water Resour., 29, 792-807, 2006.

Kollat, J. B. and Reed, P.: A framework for visually interactive decision-making and design using evolutionary multi-objective optimization (VIDEO), Environ. Model. Softw., 22, 1691-1704, 2007.

Kumar, S. V., Peters-Lidard, C. D., Tian, Y., Houser, P. R., Geiger, J., Olden, S., Lighty, L., Eastman, J. L., Doty, B., Dirmeyer, P., Adams, J., Mitchell, K., Wood, E. F., and Sheffield, J.: Land information system - an interoperable framework for high resolution land surface modeling, Environ. Modell. Softw., 21, 1402-1415, 2006.

Liu, Y. and Sun, F.: Sensitivity analysis and automatic calibration of a rainfall-runoff model using multiobjectives, Ecol. Inform., 5, 304-310, 2010.

MacLean, A. J., Tolson, B. A., Seglenieks, F. R., and Soulis, E. Multiobjective calibration of the MESH hydrological model on the Reynolds Creek Experimental Watershed, Hydrol. Earth Syst. Sci. Discuss., 7, 2121-2155, doi:10.5194/hessd-7-2121-2010, 2010. 
Madsen, H.: Automatic calibration of a conceptual rainfall-runoff model using multiple objectives, J. Hydrol., 235, 276-88, 2000.

Madsen, H.: Parameter estimation in distributed hydrological catchment modelling using automatic calibration with multiple objectives, Adv. Water Resour., 26, 205-216, 2003.

Moriasi, D., Arnold, J., Van Liew, M., Bingner, R., Harmel, R., and Veith, T.: Model evaluation guidelines for systematic quantification of accuracy in watershed simulations, T. ASABE, 50, 885900, 2007.

Morris, M. D.: Factorial sampling plans for preliminary computational experiments, Technometrics, 33, 161-174, 1991.

Muleta, M. K. and Nicklow, J. W.: Sensitivity and uncertainty analysis coupled with automatic calibration for a distributed watershed model, J. Hydrol., 306, 127-145, 2005.

Nash, J. E. and Sutcliffe, J. V.: River flow forecasting through conceptual models part I - A discussion of principles, J. Hydrol., 10, 282-290, 1970.

Nelder, J. A. and Mead, R.: A simplex method for function minimization, Comput. J., 7, 308-313, 1965.

Peters-Lidard, C. D., Houser, P. R., Tian, Y., Kumar, S. V., Geiger, J., Olden, S., Lighty, L., Doty, B., Dirmeyer, P., Adams, J., Mitchell, K., Wood, E. F., and Sheffield, J.: Highperformance Earth system modeling with NASA/GSFC's Land Information System, Innov. Sys., and Soft. Eng., 3, 157-165, 2007.

Ratto, M., Pagano, A., and Young, P.: State dependent parameter meta-modelling and sensitivity analysis, Comput. Phys. Commun., 177, 863-876, 2007.

Refsgaard, J. C. and Storm, B.: MIKE SHE, in: Computer Models in Watershed Hydrology, edited by Singh, V. J., Water Resour. Publications, Rome, Italy, 809-846, 1995.

Rosolem, R., Gupta, H. V., Shuttleworth, W. J., Zeng, X., and Gonçalves, L. G. G.: A fully multiple-criteria implementation of the Sobol' method for parameter sensitivity analysis, J. Geophys. Res. Atmos., 117, D07103, doi:10.1029/2011JD016355, 2012.

Rosolem, R., Gupta, H. V., Shuttleworth, W. J., Gonçalves, L. G. G., and Zeng, X.: Towards a comprehensive approach to parameter estimation in land surface parameterization schemes, Hydrol. Process., 27, 2075-2097, 2013.
Saltelli, A. and Annoni, P.: How to avoid a perfunctory sensitivity analysis, Environ. Modell. Softw., 25, 1508-1517, 2010.

Saltelli, A., Ratto, M., Andres, T., Campolongo, F., Cariboni, J., Gatelli, D., Saisana, M., and Tarantola, S.: Global Sensitivity Analysis, The Primer, Wiley and Sons, Chichester, UK, 2008.

Shafii, M. and De Smedt, F.: Multi-objective calibration of a distributed hydrological model (WetSpa) using a genetic algorithm, Hydrol. Earth Syst. Sci., 13, 2137-2149, doi:10.5194/hess-132137-2009, 2009.

Tang, Y., Reed, P., and Wagener, T.: How effective and efficient are multiobjective evolutionary algorithms at hydrologic model calibration?, Hydrol. Earth Syst. Sci., 10, 289-307, doi:10.5194/hess-10-289-2006, 2006.

Van Werkhoven, K., Wagener, T., Reed, P., and Tang, Y.: Sensitivity-guided reduction of parametric dimensionality for multi-objective calibration of watershed models, Adv. Water Resour., 32, 1154-1169, 2009.

Vrugt, J., Gupta, H. V., Bastidas, L. A., Bouten, W., and Sorooshian, S.: Effective and efficient algorithm for multiobjective optimization of hydrologic models, Water Resour. Res., 39, 1214, doi:1210.1029/2002WR001746, 2003.

Wöhling, T., Barkle, G. F., and Vrugt, J. A.: Comparison of three multiobjective optimization algorithms for inverse modeling of vadose zone hydraulic properties, Soil Sci. Soc. Am. J., 72, 305319, 2008

Yang, J., Reichert, P., Abbaspour, K. C., and Yang, H.: Hydrological modelling of the Chaohe Basin in China: statistical model formulation and Bayesian inference, J. Hydrol., 340, 167-182, 2007.

Yang, J.: Convergence and uncertainty analyses in Monte-Carlo based sensitivity analysis, Environ. Model. Softw., 26, 444-457, 2011.

Yapo, P. O., Gupta, H. V., and Sorooshian, S.: Multiobjective global optimization for hydrologic models, J. Hydrol., 204, 83-97, 1998

Zitzler, E, Laumanns, M., and Thiele, L.: SPEA2: improving the strength Pareto evolutionary algorithm, Tech Rep TIK-103, Department of Electrical Engineering, Swiss Federal Institute of Technology, 2001. 